Service social

\title{
L'entente cadre entre les CLSC et les centres jeunesse. Une étape dans la construction d'un continuum véritable de services aux jeunes
}

\section{Claude Larivière}

Volume 47, numéro 3-4, 1998

Enfance : enjeux et réalités 2000

URI : https://id.erudit.org/iderudit/706799ar

DOI : https://doi.org/10.7202/706799ar

Aller au sommaire du numéro

Éditeur(s)

École de service social de l'Université Laval

ISSN

1708-1734 (numérique)

Découvrir la revue

Citer cet article

Larivière, C. (1998). L'entente cadre entre les CLSC et les centres jeunesse. Une étape dans la construction d'un continuum véritable de services aux jeunes. Service social, 47(3-4), 169-187. https://doi.org/10.7202/706799ar
Résumé de l'article

Les centres jeunesse et les CLSC se sont donné une entente cadre pour faciliter leur collaboration en 1998. Une recherche effectue le suivi de ces relations renouvelées et permet de mesurer à la fois les changements de pratiques et les difficultés qu'entraîne toute modification substantielle des cultures organisationnelles. Certains facteurs comme le manque de ressources et le contexte organisationnel diminuent la capacité de concertation. Bien que des résultats intéressants soient enregistrés, les partenaires ne constituent pas encore un véritable continuum assurant l'accès souhaité aux services pour les jeunes en difficulté et leurs parents. Une telle transformation des pratiques demande du temps, des ressources suffisantes et un soutien politique, des conditions qui sont loin d'être assurées. 


\section{L'entente cadre entre les CLSC et les centres jeunesse Une étape dans la construction d'un continuum véritable de services aux jeunes}

Claude LARIVIĖRE

Les centres jeunesse et les CLSC se sont donné une entente cadre pour faciliter leur collaboration en 1998. Une recherche effectue le suivi de ces relations renouvelées et permet de mesurer à la fois les changements de pratiques et les difficultés qu'entraîne toute modification substantielle des cultures organisationnelles. Certains facteurs comme le manque de ressources et le contexte organisationnel diminuent la capacité de concertation. Bien que des résultats intéressants soient enregistrés, les partenaires ne constituent pas encore un véritable continuum assurant l'accès souhaité aux services pour les jeunes en difficulté et leurs parents. Une telle transformation des pratiques demande du temps, des ressources suffisantes et un soutien politique, des conditions qui sont loin d'être assurées.

CLSC and youth centers (centres jeunesse) gave each other an agreement to facilitate their collaboration in 1998. A research carries out the follow-up of these renewed relations and makes it possible to measure at the same time the changes of practices and the difficulties which comprises any substantial modification of the organizational cultures. Certain conditions as the lack of resources and the organizational context decrease the capacity of dialogue. Although interesting results are recorded, we are still far from a true continuum ensuring the access desired to the services for the young people in difficulty and their parents. Such a transformation of the practices requires time, resources and political support, conditions which are far from being assured. 
Les jeunes en difficulté et leur famille font principalement appel aux intervenants sociaux des CLSC (également présents en milieu scolaire) et des centres jeunesse. Selon la situation et la nature du besoin, l'intervention pertinente peut relever d'une approche préventive, utiliser les ressources de la communauté ou nécessiter une intervention protectrice et même un placement. Le niveau de collaboration entre intervenants sociaux des centres jeunesse et des CLSC constitue un enjeu important pour assurer la meilleure réponse possible. Or, jusqu'en 1998, une telle concertation n'existait qu'occasionnellement en raison du leadership local d'intervenants ou de gestionnaires innovateurs.

Soucieuses d'améliorer les relations entre les deux types d'établissements publics pour mieux répondre aux besoins d'une clientèle commune, les deux associations qui réunissent l'ensemble des CLSC et des centres jeunesse ont franchi un pas important, en juin 1998, en élaborant conjointement une entente cadre précisant les rôles respectifs et prévoyant des modalités concrètes de collaboration entre les CLSC et les centres jeunesse (CJ). En ce sens, le document Des établissements qui s'appuient pour les services aux enfants, aux jeunes et à leur famille apparaît comme un moment fort de l'histoire de la collaboration interorganisationnelle en vue d'améliorer les services aux jeunes. Le document précise un ensemble de fonctions nécessaires (promotion, prévention, intervention, coordination) et clarifie les rôles respectifs des deux partenaires à l'égard de chacune d'entre elles. En ce faisant, les CLSC et les CJ contribuent à constituer l'embryon d'un environnement favorable au développement éventuel d'un continuum de services à la jeunesse.

Les deux associations concernées ont aussi décidé d'apporter un soutien concret aux établissements pour s'assurer de l'implantation de cette entente dans toutes les régions du Québec. Elles souhaitaient éviter qu'une entente, si bonne soit-elle, ne soit pas suivie d'efforts réels des partenaires pour développer leur coopération et la traduire en modifications sensibles des pratiques.

Cette préoccupation pour le suivi du changement proposé a conduit à la mise en place d'une recherche provinciale visant à donner aux partenaires structurels (les deux associations), au 
ministère de la Santé et des Services sociaux et aux partenaires terrain (les régies régionales, les CLSC et les centres jeunesse) l'assurance que leurs efforts réunis pour la mise en œuvre du protocole précisant les conditions et les formes de collaboration attendues donneront lieu aux initiatives souhaitées par ses promoteurs et aux résultats recherchés pour le bénéfice de la clientèle. Cet article fait état des premiers résultats de cette recherche et les met en relation avec les conditions habituellement nécessaires pour qu'une coopération inter-organisationnelle soit fructueuse.

\section{LES ASPECTS MÉTHODOLOGIQUES}

Cette recherche évaluative vise à approfondir la connaissance de la dynamique des changements dans et entre des organisations professionnelles et des conséquences possibles de ceux-ci sur la clientèle. Elle repose sur la constitution d'un échantillon national de répondants (chaque établissement fournissant cinq noms par territoire de CLSC) et sur une approche longitudinale (questionnaire répété en 1999, 2000 et 2001) afin de saisir le processus d'implantation dans l'ensemble du Québec ainsi que les variations propres à chacune des régions, aux deux types d'établissements et aux deux catégories de répondants (gestionnaires et intervenants). Le choix d'une approche par territoire de CLSC s'explique par la nécessité de pouvoir comparer les perceptions des organisations qui collaborent et par le fait que les centres jeunesse ont réorganisé leurs équipes de prise en charge sur cette base (Centres jeunesse de Québec, 1997).

L'échantillon de 1999 comptait 996 répondants et le taux de réponse fut de $92,4 \%$ (921 questionnaires), quelques établissements sur les 150 sollicités ayant décidé de ne pas participer à la recherche (Larivière et Barbeau).

La recherche permet de prendre en quelque sorte le pouls d'un très grand nombre d'acteurs directement engagés dans le processus. En effet, plutôt que de rester au niveau des décideurs et des directions des établissements, l'échantillon a été construit afin de mesurer les perceptions de ceux qui doivent réaliser les changements nécessaires dans leurs pratiques quotidiennes pour que l'entente cadre devienne une réalité concrète. Le questionnaire 
était orienté vers la mise en évidence du point de départ: Quel était le vécu antérieur des répondants avec leur partenaire? Quelles sont leurs attentes à l'égard de ce que la collaboration nouvelle peut apporter à leur clientèle?

Ce premier portrait de la situation fut tracé entre mars et octobre 1999, alors que plusieurs régions commençaient à peine leurs efforts pour implanter l'entente. II sera intéressant de voir l'évolution qui se dégagera du deuxième (en 2000) et du troisième questionnaire (en 2001).

\section{DE L'INTÉRÊT À TRAVAILLER ENSEMBLE}

Antérieurement à l'entente cadre, 85,7 \% avaient déjà une expérience de collaboration avec leur partenaire, positive selon $83,3 \%$ des répondants. Quelles sont les caractéristiques de cette expérience de travail? Six formes de collaboration ressortent : la collaboration dans des cas communs et la co-intervention (33,8 \%), les comités de travail, les tables de concertation et les projets communs $(23,2 \%)$, les demandes de services provenant du partenaire $(20,4 \%)$, les échanges d'information $(11,6 \%)$, les signalements $(9,7 \%)$ et enfin les formations communes $(1,0 \%)$.

Comment qualifient-ils cette expérience de travail? Les répondants penchent très nettement pour un jugement positif $(83,3 \%)$ plutôt que négatif $(15,4 \%)$. De façon générale, les expériences de travail sont jugées plutôt positives ou positives (à $87,5 \%$ pour les cadres des CJ, à 86,5\% pour les intervenants de CJ, à 85,5\% pour les cadres des CLSC et à $80,4 \%$ pour les intervenants des CLSC), mais on note que l'expérience antérieure de travail des intervenants des CLSC avec les CJ est évaluée par eux comme ayant été négative ou plutôt négative dans $18,0 \%$ des cas.

Le questionnaire a mesuré leurs perceptions des relations avec le CLSC ou le CJ (selon le cas) de leur territoire. En 1999, ces relations sont dites assez ou très faciles selon $69,9 \%$ des répondants et assez ou très difficiles pour $25,7 \%$ d'entre eux; $4,4 \%$ disent qu'elles n'existent pas. En apparence, on ne peut pas dire qu'il y a une opposition de principes faisant obstacle à la collaboration envisagée. Mais il faut pousser l'analyse plus en détail. 
Premier constat, les cadres et les intervenants ne vivent pas la même réalité. Les gestionnaires des $\mathrm{CJ}$ trouvent leurs relations aussi faciles (à 82,5 \%) que leurs collègues des CLSC (à 79,0 \%). Mais seulement $67,4 \%$, des intervenants des CJ et $67,0 \%$ de ceux des CLSC trouvent cela assez facile ou très facile. Inversement, alors que $19,9 \%$ des intervenants $C J$ et 20,2 \% des cadres en CLSC trouvent cela assez difficile, ce pourcentage monte à $27,9 \%$ chez les intervenants des CLSC, pourcentage qui constitue l'expression d'un malaise certain.

Nous avons cherché à savoir si le fait pour un répondant d'avoir travaillé dans l'autre organisation pouvait modifier sa perception. Chez les répondants des CLSC, le fait d'avoir antérieurement travaillé en centre jeunesse ne modifie pas la perception des relations. Par contre, en $\mathrm{CJ}$, le fait d'avoir travaillé antérieurement en CLSC contribue à faciliter les relations ou à améliorer la perception de celles-ci.

\section{LA CONNAISSANCE ET L'ADHÉSION À L'ENTENTE}

Le protocole d'entente est connu de $89,4 \%$ des répondants et $43 \%$ des répondants ont participé à au moins une activité d'appropriation. Ce taux varie parfois selon le statut des répondants (cadres ou intervenants) et l'établissement, mais, de façon générale, les répondants se révèlent très favorables aux orientations générales de l'entente (à $98 \%$ ). L'adhésion aux différents éléments (définitions et mandats) est légèrement moins élevée. Deux aspects soulèvent un peu moins l'accord: l'accès propre à la réadaptation $(75,9 \%)$ et la contribution au plan du traitement prévue pour les CLSC (87,8\%). Un constat s'impose : le processus pour arriver à l'objectif poursuivi et les moyens dont on dispose pour le faire posent plus de problèmes que la fin poursuivie, relativement bien acceptée.

Globalement, les répondants pensent que les conditions de réalisation ne sont que très partiellement réunies. Ceux qui disent qu'elles existent déjà se situent à 6,8 \%. Inversement, 28,5\% des répondants disent que ces conditions n'existent pas et les autres affirment qu'elles existent partiellement (64,7\%). Cela n'étonne pas vraiment au tout début d'un processus. II faut aussi y lire un message à l'intention des dirigeants des organisations voulant 
qu'un discours de coopération ne suffise pas pour que celle-ci se concrétise.

\section{LES CONDITIONS DE L'ARTICULATION DE LA COOPÉRATION LOCALE}

Dans l'ensemble du Québec, $76,7 \%$ des répondants affirment qu'il y a eu des initiatives de coopération avec leur partenaire CJ ou CLSC; cela varie cependant selon les régions, le statut des répondants (plus de cadres que d'intervenants en ont vécu) et les établissements. Et $85,7 \%$ des répondants trouvent ces initiatives intéressantes ou prometteuses.

De plus, $53,7 \%$ des répondants envisagent de prendre une initiative en vue de réaliser l'un ou l'autre des éléments de l'entente cadre. Le taux peut toutefois varier du simple au double selon que le répondant est un intervenant ou un gestionnaire, le gestionnaire étant plus proactif. Des variations significatives existent selon les régions.

Interrogé sur les conditions nécessaires à la réussite de l'entente, l'échantillon de répondants fournit une liste particulièrement riche de conditions à réunir. Les premières portent sur le développement d'une conception partagée, alors que les secondes regroupent des moyens pour l'actualiser. Examinons-les.

\section{LE DÉVELOPPEMENT D'UNE CONCEPTION PARTAGÉE}

Les répondants soulignent qu'une réelle conception commune doit exister avant que la collaboration se développe sur le terrain et dans les pratiques quotidiennes. Les principes mis en avant, comme se centrer sur les besoins des clients, partager une vision commune des besoins, des ressources et des priorités, reconnaître les expertises de chacun et développer des approches communes pour l'évaluation, inspirent les nouvelles pratiques. Mais il faut aussi un positionnement clair des gestionnaires, c'està-dire un engagement des directions générales et le soutien des cadres opérationnels à qui l'on doit accorder une marge de manœuvre suffisante pour qu'ils puissent prendre des initiatives.

Les notions de volontariat des clients en CLSC et la définition du concept de protection par les centres jeunesse posent des pro- 
blèmes à leur partenaire. Pour les CLSC, un client volontaire doit formuler sa propre demande; pour les CJ, bien des situations qui apparaissent suffisamment détériorées pour nécessiter une prise en charge légale du point de vue du CLSC ne répondent pas aux critères de compromission de la DPJ. Cela crée de nombreuses frustrations. Par ailleurs, le fait de mieux connaître les critères d'admissibilité aux services du partenaire réduit sensiblement le risque d'une fausse attente.

Plusieurs considèrent que la participation à des formations communes sur des questions comme l'évaluation, les plans de services individualisés, le placement et la concertation contribue non seulement à rapprocher les personnes en leur permettant de mieux comprendre le point de vue de l'autre, mais assure aussi les organisations que les mêmes informations sont données à ceux qui devront ensuite collaborer. Des progrès en ce sens sont perceptibles, même si beaucoup reste à faire pour y parvenir en raison de cultures professionnelles différentes.

\section{LES MOYENS D'ACTUALISER DES PRATIQUES CONCERTÉES}

Sur le plan des moyens pour actualiser cette orientation commune, une longue liste peut être dressée. Des répondants soulignent l'importance de définir plus clairement les mandats, les missions et les rôles des deux types d'établissements, ce qui laisse entendre qu'il subsiste à leurs yeux des zones grises qui créent de la confusion ou génèrent des interprétations divergentes, malgré les efforts des rédacteurs de l'entente cadre pour tenter d'être aussi précis que possible.

Autre aspect clé, le fait de ne pas disposer des ressources financières et humaines suffisantes pour les deux types d'établissements, mais plus particulièrement pour les CLSC. Dans le cas des centres jeunesse, il s'agit surtout d'alléger la charge de travail pour permettre aux intervenants de s'impliquer davantage avec leur partenaire. 
II faut aussi des lieux d'échange et de concertation pour améliorer la connaissance réciproque des intervenants. Évidemment, participer à des comités mixtes, aux tables de discussion et de concertation, à des groupes de travail nécessite d'abord que les intervenants disposent de temps à cette fin, ce qui n'est pas toujours le cas étant donné les listes d'attente et les charges de cas.

Les formations communes apparaissent importantes pour se donner une information partagée, une connaissance des rôles respectifs, de même que des outils diagnostiques et de traitement communs ou, du moins, complémentaires.

Comme il y aura toujours des situations litigieuses, il faut absolument se donner une forme de mécanisme pour les traiter. II peut s'agir d'agents de liaison qui essaient de clarifier ce qui doit être fait et qui en appellent, au besoin, aux gestionnaires responsables des équipes territoriales ou à un comité régional ou sous-régional qui se réunit lorsque des questions méritent son attention.

Des répondants souhaitent la mise en commun des données clientèles, ce qui suppose que l'ensemble des organisations possèdent des systèmes informatiques capables de communiquer en réseau. Évidemment, une telle approche pose aussi la question des barrières à mettre en place pour assurer la confidentialité et pour limiter l'accès à cette information.

Les intervenants demandent aussi un meilleur encadrement clinique pour les rassurer lorsqu'ils sont aux prises avec des cas complexes et des problématiques nouvelles. L'ouverture sur l'autre partenaire présuppose souvent une polyvalence accrue et réduit d'autant la possibilité de se concentrer auprès d'un seul type de clients pour lequel on possède une plus grande expertise. Cet encadrement peut prendre la forme de pairs expérimentés désignés comme répondants qui peuvent aider les jeunes praticiens ou ceux qui doivent faire face à des difficultés nouvelles, d'adjoints cliniques ou de ressources expertes qui peuvent être consultés au besoin.

Malgré la multiplication des événements et des communiqués pour faire connaître l'entente cadre et ses modalités d'application, 
de nombreux répondants insistent pour que l'on se préoccupe de l'efficacité des communications afin que le message rejoigne véritablement les intervenants et qu'il soit bien compris.

Des répondants rappellent la nécessité d'investir davantage dans la prévention et la promotion afin d'éviter la détérioration des situations, craignant que tous les efforts convergent vers le soulagement des listes d'attente et les cas les plus criants.

Une fois que des situations signalées sont retenues, les répondants des centres jeunesse souhaitent qu'on instaure une période tampon avant de fermer le dossier en CLSC, pour leur permettre de planifier la continuité de la prise en charge, si celle-ci s'avère nécessaire.

Plusieurs reconnaissent la nécessité d'accroître le nombre de plans d'intervention et de plans de services individualisés, lorsque la situation le demande. L'idée qu'une bonne intervention commande des objectifs clairement établis semble bien acceptée; le niveau de formalisation de ces objectifs reste à définir pour éviter de tomber dans la bureaucratie inutile.

Les répondants proposent de mettre en place des mécanismes de référence personnalisés afin que le lien se fasse naturellement entre l'accueil du CLSC et l'équipe de réception et de traitement des signalements du Centre jeunesse, dans un sens ou dans l'autre.

On propose enfin la mise en place éventuelle d'un continuum de services intégrés pour pousser beaucoup plus loin la coopération entre CLSC et CJ et, surtout, pour l'élargir à l'ensemble des partenaires du milieu, qu'ils soient communautaires ou institutionnels, du réseau de la santé et des services sociaux ou des autres secteurs (justice, éducation, municipalités, loisirs).

\section{LES PRATIQUES COURANTES ET EN ÉMERGENCE}

La collaboration actuelle varie beaucoup d'un établissement à l'autre, parfois même d'un point de services à l'autre. Certaines pratiques sont tout de même largement répandues, alors que d'autres moins courantes se développent. 
Ainsi, trois pratiques sont considérées comme courantes, bien que de façon variable selon les territoires. II s'agit des demandes de services (signalement, transfert de dossiers, placement), des échanges et des discussions de cas lors de demandes de services de part et d'autre, puis des tables et des projets de concertation. Ces pratiques peuvent être considérées comme le noyau de base, assez traditionnel, qu'il faut certainement conserver, mais également chercher à dynamiser et à élargir.

Les pratiques qui se développent correspondent davantage à ce qui est souhaité pour modifier concrètement les façons de faire et pour assurer la continuité des services aux jeunes. Ainsi, plusieurs répondants sont fiers de mener des interventions communes ou en complémentarité dans des dossiers communs. Cela se traduit notamment par l'élaboration en commun de plans d'intervention et de plans de services individualisés. La collaboration entre les intervenants ne se limite plus aux seuls intervenants sociaux, puisqu'on rapporte également plusieurs cas de co-intervention entre les infirmières en périnatalité et les éducateurs externes d'un centre jeunesse. II y a de plus en plus d'échanges d'expertise, ce qui constitue une forme de reconnaissance explicite de l'apport spécifique de chacun des partenaires. Cela s'ajoute aux tables d'accès et aux comités de placement qui permettent de discuter des besoins perçus pour certains jeunes.

Les partenaires tentent aussi de mettre en place des mécanismes de référence privilégiés et des transferts personnalisés. Pour faciliter cela, des agents de liaison sont chargés d'acheminer les demandes et les références des centres jeunesse aux bons services et aux bonnes personnes en CLSC.

Enfin, les contributions occasionnelles des deux partenaires permettent l'animation de groupes destinés aux jeunes et aux parents. La participation aux programmes de prévention et d'information (sur les gangs, les abus sexuels, etc.) complète ces activités de nature préventive.

\section{DE NOMBREUSES DIFFICULTÉS RESTENT ENCORE À SURMONTER}

Cette amplification marquée des activités qui permettent de combiner l'apport des intervenants des deux partenaires ne signifie 
pas pour autant que les difficultés sont inexistantes. Tout au contraire, elles sont presque partout présentes, selon des intensités variables, mais les partenaires s'en soucient et tentent généralement de trouver des solutions. Voilà peut-être un changement majeur apporté par l'entente cadre.

Voici maintenant les difficultés recensées comme étant les plus importantes. II y a d'abord un problème majeur autour de la question du manque de personnel et de la surcharge perçue de travail. Vue par des intervenants des CLSC comme un surplus de tâches, quelque chose qu'il faut faire en plus, sans ressources supplémentaires l'entente peut déranger plutôt que de faciliter leur travail. Dans les centres jeunesse, la taille actuelle des charges de cas (autour de 25 par intervenant, parfois 28 , alors que l'idéal se situe à moins de 20) rend difficile l'ajout de rencontres avec les partenaires du CLSC où l'intervenant varie selon les cas.

Sur le plan des processus, les délais de réponse aux demandes et dans les transmissions d'informations sont signalés comme constituant une difficulté, tout comme le fait que les intervenants ne retournent pas toujours leurs appels. S'ajoute à cela une certaine confusion des rôles et des mandats en raison de l'incompréhension du rôle des intervenants CJ par les intervenants CLSC et des cultures organisationnelles différentes.

Cela se reflète parfois par des perceptions différentes des problèmes ou encore par une interprétation différente de la notion de volontariat par rapport à l'approche proactive demandée aux CLSC. Les praticiens des CLSC craignent la perte de la spécificité de leur travail en CLSC que pourrait constituer la prise en charge de cas en provenance des CJ. Ils soulignent que leurs partenaires ne les informent pas souvent du cheminement de leur demande et qu'ils ont de la difficulté à comprendre les motifs de rétention ou de non-rétention de leurs signalements.

Il faut se rappeler qu'une partie significative des intervenants sociaux des CLSC ont déjà travaillé dans des centres de services sociaux (CSS) et ont choisi de ne plus faire carrière en contexte d'autorité. Certains répondants disent carrément craindre d'avoir à changer leur méthode de travail et de perdre ainsi leur identité d'intervenants sociaux de CLSC. Ce sont souvent les mêmes qui 
croient que les CJ se sont approprié une partie du mandat du CLSC en développant l'intervention de milieu des éducateurs.

II ne faut pas s'étonner que, lorsque surgit une confusion sur les rôles ou les responsabilités des partenaires, ceux-ci adoptent une attitude défensive et se raidissent, ce qui conduit à un manque de concertation dans des dossiers communs.

Malgré ces difficultés réelles, 68,2 \% des répondants croient qu'il faut élargir cette entente à d'autres partenaires préoccupés par les besoins des jeunes. Invités à préciser lesquels, ils indiquent principalement des partenaires institutionnels (milieu scolaire, pédopsychiatrie, services policiers, etc.) et, seulement ensuite, les organismes communautaires.

\section{LE CONTEXTE ORGANISATIONNEL INFLUENCE LA COLLABORATION}

Une recherche de Glisson et Hemmelgarn (1998) a fait ressortir l'impact du climat organisationnel sur la qualité des services et la capacité des organisations à bâtir de bonnes relations interorganisationnelles. Quatre questions furent alors introduites pour mieux comprendre le contexte dans lequel se vit le travail quotidien de ceux et de celles à qui l'on demande de se concerter. L'interprétation de ces données doit cependant être prudente, puisque seul un petit échantillon de l'ensemble du personnel des CJ et des CLSC (soit 921 personnes) exprime ici ses perceptions.

La première question portait sur le style de gestion. Quatre choix étaient proposés : deux styles traditionnels, soit le style contrôlant (approche néo-taylorienne) et le style bureaucratique (approche technobureaucratique), et deux styles plus adaptés aux besoins actuels, soit le style innovateur (approche de l'excellence) et le style supportant (approche de la contingence). Larivière (1995) mettait en évidence que les styles de gestion des 11 CLSC étudiés étaient à peu près répartis également entre les deux premiers et les deux derniers. Les 822 répondants de 1999, provenant de l'ensemble du Québec, en regroupant à la fois les intervenants et les gestionnaires des CLSC et des CJ qui ont accepté de préciser leur perception, donnent un résultat assez semblable. En effet, $40,8 \%$ choisissent le style de gestion bureaucratique pour décrire la dominante de leur organisation, $27,5 \%$ un style de gestion 
supportant, $15,8 \%$ un style innovateur et 9,5 \% un style contrôlant. Cela signifie que les styles traditionnels recueillent $50,3 \%$ des choix, alors que les styles novateurs en reçoivent $43,3 \%$. La différence entre ces résultats et l'ensemble des répondants (100\%) vient de ce que $6,4 \%$ ne savent pas faire un choix; cela s'explique par leur peu d'expérience dans l'organisation, leur ambivalence ou la difficulté de choisir un seul terme pour désigner le style de gestion de l'ensemble de l'organisation.

Rappelons qu'une gestion souple signifie plus de marge de manœuvre pour les gestionnaires de premier niveau et leurs intervenants. Différentes expériences menées dans des centres jeunesse montrent la possibilité d'autonomiser davantage les équipes et leurs membres (Centre jeunesse de l'Estrie, 1998), bien que cela nécessite d'abord l'existence de conditions favorables qui ne sont pas faciles à réunir.

II y a toutefois des variations importantes selon le type d'établissement et le statut des répondants. Ainsi, les gestionnaires et les intervenants des CJ s'entendent pour considérer le style de gestion bureaucratique comme le plus important au sein de leur organisation, même si cette importance semble beaucoup plus grande du point de vue des intervenants $(63,9 \%$ contre $48,3 \%)$. II existe un consensus sur l'importance du style de gestion axé sur le contrôle en $\mathrm{CJ}$, que les répondants soient gestionnaires ou intervenants.

En CLSC, les perceptions des gestionnaires et des intervenants divergent sensiblement. Les gestionnaires se définissent comme étant préoccupés par le soutien à leurs intervenants $(47,0 \%)$ et innovateurs $(39,1 \%)$, ces deux styles représentant ensemble $86,1 \%$ des choix exprimés. Les intervenants reconnaissent l'importance du style supportant dans les CLSC, auquel ils accordent $32,1 \%$ de leurs choix, mais ils mettent d'abord en valeur le caractère bureaucratique de leur organisation (36,0\%).

La deuxième question de la partie sur le contexte organisationnel portait sur le climat de travail de leur organisation. Les résultats obtenus traduisent sans doute la perception que les répondants ont de leur fardeau de tâche tout autant que du climat dans lequel s'effectue ce travail: décrit comme assez stressant par $45,1 \%$ 
des répondants, le climat de travail apparaît assez stimulant pour $21,0 \%$ des répondants, agréable pour $17,9 \%$ et pénible pour $12,9 \%$ d'entre eux; notons aussi que $3 \%$ ne se prononcent pas.

Globalement, $58 \%$ des répondants trouvent insatisfaisant le climat de travail de leur organisation, alors que 38,9\% estiment que cela va bien. Tant en CJ qu'en CLSC, la catégorie " assez stressant " est choisie par le plus grand nombre de répondants. Les intervenants des deux types d'organisations sont plus nombreux que leurs gestionnaires à percevoir le climat de travail comme pénible. Rappelons que dans une recherche sur l'impact des transformations sur les travailleurs sociaux Bernier et Larivière (1996) ont souligné leur niveau élevé de détresse psychologique mesuré avec l'échelle de Santé Québec. Les changements affectent beaucoup les personnes qui les vivent et un climat détérioré rend plus difficile l'introduction d'autres changements, comme l'expérience des autorisations confiées aux éducateurs par les Centres jeunesse de Montréal l'a démontré (Larivière et Boisvert, 2000).

La troisième question invitait les répondants à dire si leur organisation leur apparaît efficace en leur fournissant les moyens de réaliser leur tâche. La catégorie qui réunit le plus de choix est plutôt efficace, avec $54,2 \%$ des répondants, puis plutôt inefficace, avec $32,2 \%$, très inefficace $(4,9 \%)$ et très efficace $(4,3 \%)$, alors que $4,4 \%$ ne savent pas laquelle choisir.

Dans l'ensemble, les répondants perçoivent donc leur organisation comme étant plus efficace $(58,5 \%)$ qu'inefficace $(37,1 \%)$; cependant, les intervenants des CJ et des CLSC sont plus nombreux que les gestionnaires à considérer leur organisation comme étant peu efficace (écart d'environ $20 \%$ dans les deux cas). Ainsi, les intervenants des CJ la disent inefficace dans 52,8\% des cas comparativement à $34,4 \%$ pour leur supérieur immédiat. En CLSC, $34,9 \%$ des intervenants trouvent leur organisation inefficace contre seulement $10,3 \%$ de leurs supérieurs qui partagent cette opinion.

La dernière question invitait les répondants à exprimer leur degré de satisfaction au travail. Les données sont un peu plus positives, puisque $62,5 \%$ des répondants se disent satisfaits, $23,1 \%$ insa- 
tisfaits, $9,4 \%$ très satisfaits, $2,9 \%$ très insatisfaits, alors que seulement $2,1 \%$ n'ont pas d'opinion.

Si les répondants sont majoritairement satisfaits de leur travail $(71,9 \%)$, les gestionnaires se disent pour leur part satisfaits ou très satisfaits au travail, soit à $83,1 \%$ en CJ et à $88,1 \%$ en CLSC. Les intervenants expriment toutefois plus d'insatisfaction que les cadres, surtout en CJ (39,4\% d'insatisfaits). Évidemment, le personnel du réseau est d'abord motivé par son action auprès de la clientèle. Mais si l'accroissement du fardeau de tâche objectif (les charges de cas) et subjectif (les difficultés professionnelles et personnelles éprouvées avec un pourcentage important de clients) n'est pas compensé rapidement par un accroissement des ressources et du soutien professionnel, un processus comme l'entente CLSC/CJ ne pourra améliorer sensiblement la qualité et la continuité des services aux jeunes et à leur famille.

\section{VERS UN CONTINUUM INTÉGRÉ DE SERVICES?}

L'entente cadre permet d'améliorer sensiblement le contexte dans lequel se situent les relations entre les CLSC et les centres jeunesse sans pour autant assurer que les changements souhaités se produisent. Le choix de bâtir une approche milieu fait par les centres jeunesse (Centres jeunesse de Montréal, 1997) ne doit pas demeurer une intention théorique. Dans les faits, nous sommes encore très loin de la mise en place d'un véritable continuum de services intégrés, bien qu'il existe sur un certain nombre de territoires des expérimentations intéressantes, comme l'hébergement d'intervenants CLSC ou CJ dans les locaux du partenaire, les équipes menant un pourcentage important de co-interventions, placées sous un gestionnaire commun CJ/CLSC ou intégrées. Mais il s'agit là d'exceptions.

La notion de continuum de services renvoie à la fois à la continuité et à la complémentarité des services demandés par une clientèle. L'idée même d'un continuum de services s'appuie sur un processus normal de réponses qui commence par une évaluation, qui passe par un traitement et qui prévoit des mesures de réinsertion, de suivi ou de soutien, selon le cas. L'accès aux services et leur coordination ressortent alors comme des enjeux clés de tout continuum. O'Brien et Bushnell (1980) ont décrit dix caractéristiques 
normatives qui permettent le plein développement d'un tel réseau de services:

1) l'accès aux services pour la clientèle (par un guichet unique, une ligne téléphonique continue ou, inversement, par une multitude de points de services de divers types mais reliés entre eux);

2) l'information et l'orientation vers le service approprié;

3) la responsabilité (confiée à une organisation ou partagée) pour le maintien, le développement et l'évaluation du réseau;

4) l'existence de protocoles définissant les contributions réciproques;

5) la mise en place d'un système commun d'information de gestion sur les services à la clientèle;

6) l'établissement de liens entre les programmes de services;

7) la tenue d'événements assurant une visibilité au réseau de services;

8) les mécanismes de résolution des conflits;

9) les mécanismes pour les plaintes des usagers;

10) les canaux permettant de recevoir le feed-back du public sur le réseau de services.

Si certaines de ces caractéristiques normatives existent déjà à des degrés divers, d'autres restent tout à fait embryonnaires ou sont inexistantes. Ainsi, le protocole élaboré par les deux associations correspond entièrement à la quatrième exigence, dans la mesure où il est mis en application. L'accès facilité aux services, l'information adéquate, l'orientation de la clientèle vers le service le plus approprié, tout comme la mise en place d'un mécanisme de résolution des conflits, sont autant d'aspects sur lesquels des progrès furent accomplis, à des degrés très variables selon les territoires. Des travaux se poursuivent sur le plan provincial pour faciliter la mise en place d'un système commun d'information; les principales difficultés à surmonter sont bien entendu la question 
de la confidentialité et celle causée par les systèmes informatiques différents et peu compatibles.

La tenue en novembre 2000 d'un congrès réunissant CLSC et CJ a certainement montré que la tenue d'événements assurant une visibilité à leurs efforts de coopération devait être renforcée et poursuivie régionalement. Quant à l'établissement de liens entre les programmes de services, il s'agit davantage d'une étape à venir à mesure que l'expérience de la collaboration autour de projets ponctuels et de la co-intervention donnera le goût aux intervenants d'acquérir une plus grande coordination de leurs efforts planifiés.

Le principal constat est certainement que la mise en place d'un tel niveau de collaboration demande de la volonté et du temps. Or, les dirigeants politiques veulent souvent des effets immédiats lorsqu'il est question de liste d'attente et de personnes sans services. Si l'environnement politique optait pour la facilité et imposait des processus forcés d'intégration, les acquis de la collaboration des dernières années risqueraient d'être perdus. L'esprit même de l'entente cadre n'est pas de transférer des ressources ou de forcer leur intégration institutionnelle; tout au contraire, elle vise d'abord et avant tout à assurer aux jeunes qu'ils recevront les services rendus nécessaires par leur état et disponibles de la part du partenaire possédant l'expertise.

Rappelons que l'approche de gestion du ministère de la Santé et des Services sociaux considère encore chaque établissement comme une entité autonome qui doit équilibrer son budget, gérer et réduire sa liste d'attente, éviter les débordements à l'urgence, maintenir un niveau acceptable de performance avec des ressources limitées, etc. II n'y a donc pas de reconnaissance ni de récompense explicites des conduites de concertation; cela peut même contribuer à abaisser le niveau reconnu de performance d'un établissement en réduisant le nombre d'unités de mesure produites par son personnel, les activités de concertation n'étant pas comptabilisées.

II faudra voir comment la situation évoluera sur le terrain au cours des deux prochaines années. Sur le plan clinique, les deux associations d'établissements collaborent pour que des outils diagnostiques communs et des sessions de formation offertes au personnel des 
CLSC et des CJ facilitent le dialogue entre les praticiens. La recherche menée avec la participation de quatre régions (AbitibiTémiscamingue, Laval, Montérégie, Québec) et soutenue par le Ministère devrait aussi permettre d'identifier les milieux où la collaboration réussit davantage et les conditions à réunir pour $y$ parvenir. Mais ces réalisations ne doivent pas faire oublier que la situation demeure toutefois fragile en raison du manque de ressources et des pressions politiques constantes pour limiter les listes d'attente.

Les gestionnaires doivent composer avec une double demande d'effectuer et de soutenir ces changements des pratiques tout en parvenant à réduire les listes d'attente. Or, ils savent qu'ils seront évalués sur leur gestion du flux de services afin d'éviter débordements, plaintes et crise. Ils choisissent donc implicitement d'investir leurs efforts dans la gestion du quotidien plutôt que dans le développement de pratiques à venir.

Les premiers résultats montrent qu'il n'y a pourtant pas de résistance véritable à surmonter et que, si un minimum de conditions sont réunies (formation préalable, information adéquate, instrumentation des pratiques nouvelles, mécanisme de règlement des différends, soutien organisationnel du changement), le changement proposé devient rapidement significatif et mobilisateur.

Évidemment, un écart considérable sépare les milieux dynamiques qui expérimentent de ceux qui n'ont pas encore entrepris d'expérimenter des formes différentes de travailler. Curieusement, des îlots innovateurs existent à proximité de milieux plutôt réfractaires, au sein d'une même région. Dans certains cas, la collaboration active dépasse le noyau de base constitué par les CLSC et les CJ et s'étend même aux organismes communautaires jeunesse ou à d'autres partenaires institutionnels. Mais cette tendance à l'ouverture vers un continuum plus intégré de services aux jeunes reste encore plus un souhait qu'une réalité.

Claude LARIVIËRE Professeur agrégé École de service social Université de Montréal 


\section{Références bibliographiques}

ASSOCIATION DES CENTRES JEUNESSE DU QuÉBEC et ASSOCIATION DES CLSC ET DES CHSLD DU QUÉBEC (1998). Des établissements qui s'appuient pour les services aux enfants, aux jeunes et à leur famille, Montréal.

BeRnieR, D. et C. LARIVIERE (1996). Soutien organisationnel aux pratiques d'intervention en contexte de changement, Montréal, École de service social, Université de Montréal.

CentRes JeUnesse DE L'ESTRIE (1998). Pour des services de qualité aux jeunes en difficulté, à leurs parents et famille, Sherbrooke.

CENTRES JEUNESSE DE MONTRÉAL (1997). Les transformations liées à l'approche " milieu ». Projet collectif des Centres jeunesse de Montréal adopté au conseil d'administration du 27 mai.

CentRES JeUnesse DE QUÉBEC (1997). Distribution des services des Centres jeunesse de Québec sur base sous-régionale, Québec.

Glisson, C. et A. Hemmelgarn (1998). "The effects of organizational climate and interorganizational coordination on the quality and outcomes of children's services », Child Abuse and Neglect, vol. 22, $\mathrm{n}^{\circ} 5$, p. 401-421.

LARIVIĖRE, C. (1995). Styles de gestion, satisfaction au travail et efficacité organisationnelle perçue dans 11 CLSC. Thèse de doctorat, Département de sociologie, Université de Montréal.

LARIVIĖRE, C. et C. BARBEAU (2000). État de la collaboration entre les partenaires CLSC et les Centres jeunesse au cours de la première année (1999) à travers le Québec, Montréal, Association des Centres jeunesse du Québec et Association des CLSC et des CHSLD du Québec.

LARIVIĖRE, C. et J.-C. BOISVERT (2000). Le processus visant à confier des autorisations à des éducateurs et éducatrices des Centres jeunesse de Montréal, Montréal, Institut de recherche sur le développement social des jeunes.

O'BRIEN, G. et J. BUSHNELL (1980). "Interorganizational behavior ", dans S. Feldman (dir.), The administration of mental health services, Springfield (III.), Charles Thomas. 
